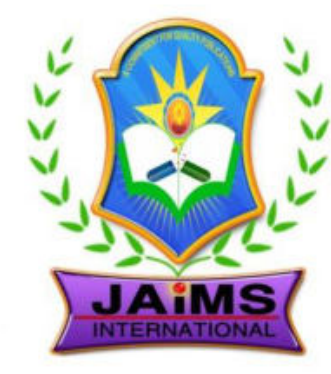

ISSN 2456-3110

Vol $5 \cdot$ Issue 1

Jan-Feb 2020

Journal of

Ayurveda and Integrated Medical Sciences

www.jaims.in

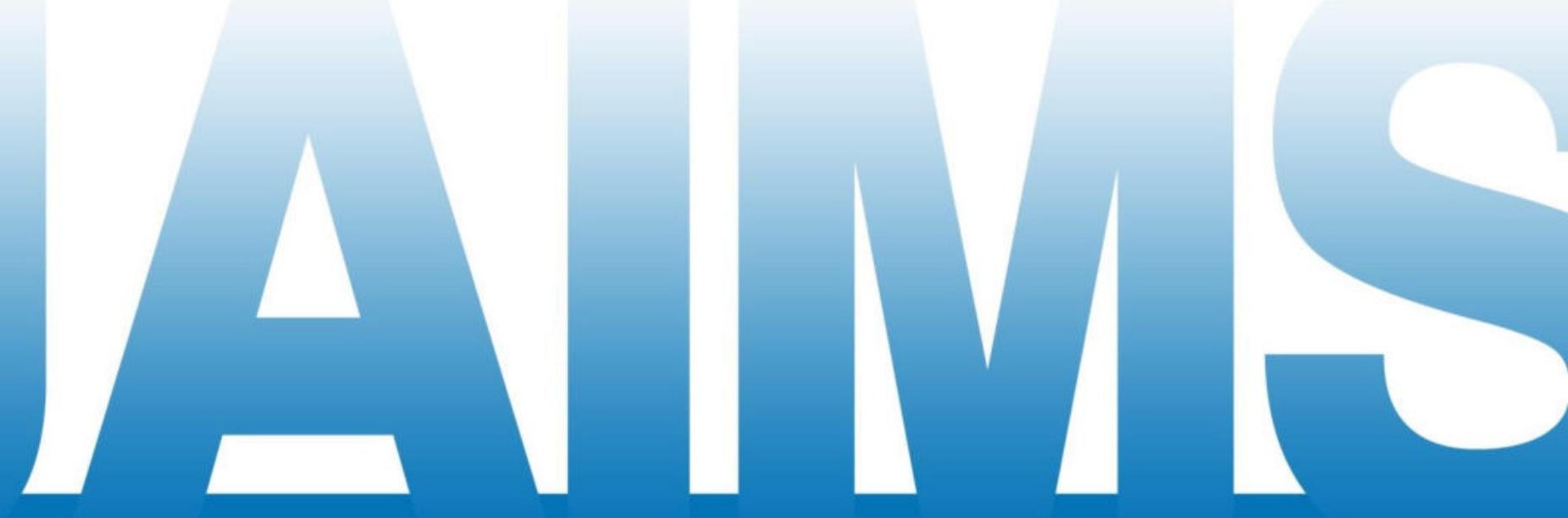

An International Journal for Researches in Ayurveda and Allied Sciences

\title{
Charaka
}




\title{
Ayurveda and Integrated Medical Sciences
}

\section{A study on the status of Kostha in Sravana and Bhadrapada Masa in different Prakruti}

\author{
Divyasree $\mathbf{C H}^{1}$, Sunil Govind Kahalekhar ${ }^{2}$, Rashmi NM ${ }^{3}$
}

${ }^{1}$ Assistant Professor, Department of Shareera Kriya, Sri Adi Siva Sadguru Alli Saheb Sivaaryula Ayurvedic Medical College, Guntakal, Andhra Pradesh, ${ }^{2}$ Professor, ${ }^{3}$ Associate Professor, Department of Shareera Kriya, Ashwini College of Ayurveda, Tumkur, Karnataka, INDIA.

\section{A B S TR A C T}

\begin{abstract}
The Prakriti is the innate constitution of an individual based on the predominance of Dosha determined at the time of conception which cannot be changed from birth till death. Qualities of Dosha are expressed on body due to its predominance and it is called Deha Prakriti. Koshta refers to the state of the Abdomen or Alimentary tract, and usually is determined by the behavior of the bowel habits. Koshta also varies according to different Prakruti. Koshta concepts helps to understand find out types of Vyadhi and its help for treatments because drug selection depends on Koshta. Sravana \& Bhadrapada Masa constitutes Varsha Rutu. So this study is an attempt to understand the relation between Koshta and Prakruti in different Kala with special reference to Sravana \& Bhadrapada Masa.
\end{abstract}

Key words: Prakruti, Kostha, Sravana, Bhadrapada Masa.

\section{INTRODUCTION}

Prakriti is the innate constitution of an individual based on the predominance of Dosha determined at the time of conception which cannot be changed from birth till death, ${ }^{[1]}$ the qualities of Dosha are expressed on body due to its predominance and it is called Prakriti of Deha. ${ }^{[2]}$

Vagbhatta has mentioned depending upon the Dosha that is predominant in the Shukra and Shonita at the time of union, the food and activities of the pregnant women, uterus and season determines the Prakriti. ${ }^{[3]}$

\section{Address for correspondence:}

\section{Dr. Divyasree $\mathrm{CH}$}

Assistant Professor, Department of Shareera Kriya, Sri Adi Siva

Sadguru Alli Saheb Sivaaryula Ayurvedic Medical College,

Guntakal, Andhra Pradesh, INDIA.

E-mail: sree02.ds@gmail.com

Submission Date: 16/01/2020

Accepted Date: $23 / 02 / 2020$

Quick Response Code

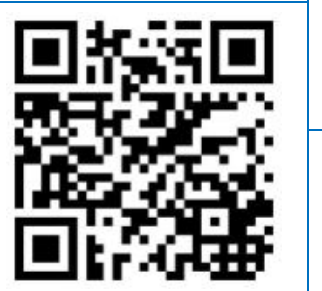

Prakriti is an expression of one's own constitution. ${ }^{[4]}$ Acharya has mentioned seven types of Prakriti. Three types with the predominance of single Dosha, three types with the predominance of two Dosha, one with the predominance of all three Doshas. ${ }^{[5]}$

Koshta refers to the state of the Abdomen or Alimentary tract, and usually is determined by the behavior of the bowel habits. Koshta varies according to different Prakrutis. ${ }^{[6]}$ For example: Vata Prakruti individual will be having Krura Koshta and Pitta Prakruti individual will be having Mrudu Koshta and Kapha Prakruti individual will be having Madhyama Koshta. ${ }^{[7]}$ When three Doshas are in equal proportion, the type of Koshta is Sama.

Rutucharya is a unique concept explained in Ayurveda with aim to combat season influence leading to disease, the proper understanding of Rtulakshanas, its impact on the status of Agni, Bala, Dosha of living being is must for the proper application of the diet \& regimen which were explained in the context of Rutucharya. Two Masa constitutes one Rutu, ${ }^{[8]}$ Sravana \& Bhadrapada Masa constitutes Varsha Rutu $^{[0]}$ and other opinion is that Nabhas \& Nabhasya Masa constitutes Varsha Rutu. ${ }^{[10]}$ During this period 
Earth is full of water, all the Disha are Vata Karaka many insects \& worms take birth in this Rutu. Beauty of earth can be felt through the greenery vegetative, smaller waterfalls and muddy earth lakes look like sea the demarcation of land area and area of water are not clear because of abundant water in this Rutu the tender plants attains good strength and the plants which are dried up because of previous Rutu starts growing well, the rivers are filled with water and by the force of trees the banks are uprooted as a result in shedding of leaves, draining of water from small rivers in all directions. Vegetations are tender having least potency and Klinnata in living beings due to rain, the clouds move slowly making the sky appear dirty and the rain comes with clouds in the sky. ${ }^{[11]}$

\section{Objectives Of the Study}

1. To evaluate status of Kostha in Sravana and Bhadrapada Masa in different Prakruti of individuals.

2. To understand the relation between Prakruti and Kostha.

\section{Materials ANd Methods}

The samples who fulfilled the inclusion and exclusion criteria were selected irrespective of Sex, Religion and Marital status, 150 individuals were selected from Ashwini Ayurvedic Medical College, Hospital \& Research Centre, Tumkur and their responses about the Status of Kostha in Sravana and Bhadrapada Masa in different Prakruti was taken through questionnaire method of collecting data and then analyzed by use of desired statistical method.

\section{Inclusion Criteria}

- 150 Healthy individuals of age group of 18 to 30 years irrespective of gender.

\section{Exclusion Criteria}

- Those individuals who are suffering from chronic diseases, systemic disorders, congenital anomalies.

- Individuals of age group below 18 years.

- Individuals of age group above 30 years.
- Those who are not willing to participate in the study.

\section{About questionnaire}

First standard questionnaire was used to assess the Prakruti of an individual. There after status of Kostha was assessed with the help of the self-graded questionnaire in Sravana and Bhadrapada Masa by distributing to minimum of 150 individuals of either gender were selected from Ashwini Ayurvedic Medical College, Hospital \& Research Centre, Tumkur for the purpose. After collection of data the response was analysed with suitable statistical method.

\section{Results}

Table 1: Status of Kostha in different Prakruti.

\begin{tabular}{|c|c|c|c|c|c|c|}
\hline & & & \multicolumn{3}{|l|}{ Kostha } & \multirow{2}{*}{$\begin{array}{l}\text { Tot } \\
\text { al }\end{array}$} \\
\hline & & & $\begin{array}{l}\text { Krura } \\
\text { Kostha }\end{array}$ & $\begin{array}{l}\text { Madhya } \\
\text { ma } \\
\text { Kostha }\end{array}$ & $\begin{array}{l}\text { Mrudu } \\
\text { Kostha }\end{array}$ & \\
\hline \multirow[t]{7}{*}{$\begin{array}{l}\text { Pra } \\
\text { kriti }\end{array}$} & \multirow[t]{3}{*}{$\begin{array}{l}\text { Vatapra } \\
\text { dhana }\end{array}$} & $\begin{array}{l}\text { Cou } \\
\text { nt }\end{array}$ & 21 & 18 & 11 & 50 \\
\hline & & $\begin{array}{l}\text { Exp } \\
\text { ecte } \\
d \\
\text { Cou } \\
\text { nt }\end{array}$ & 17.7 & 21.7 & 10.7 & $\begin{array}{l}50 . \\
0\end{array}$ \\
\hline & & $\begin{array}{l}\% \text { of } \\
\text { Tota }\end{array}$ & $14.0 \%$ & $12.0 \%$ & $7.3 \%$ & $\begin{array}{l}33 . \\
3 \%\end{array}$ \\
\hline & \multirow[t]{3}{*}{$\begin{array}{l}\text { Pittapra } \\
\text { dhana }\end{array}$} & $\begin{array}{l}\text { Cou } \\
\text { nt }\end{array}$ & 15 & 24 & 11 & 50 \\
\hline & & $\begin{array}{l}\text { Exp } \\
\text { ecte } \\
\text { d } \\
\text { Cou } \\
\text { nt }\end{array}$ & 17.7 & 21.7 & 10.7 & $\begin{array}{l}50 . \\
0\end{array}$ \\
\hline & & $\begin{array}{l}\% \text { of } \\
\text { Tota } \\
\text { I }\end{array}$ & $10.0 \%$ & $16.0 \%$ & $7.3 \%$ & $\begin{array}{l}33 . \\
3 \%\end{array}$ \\
\hline & $\begin{array}{l}\text { Kaphapr } \\
\text { adhana }\end{array}$ & $\begin{array}{l}\text { Cou } \\
\text { nt }\end{array}$ & 17 & 23 & 10 & 50 \\
\hline
\end{tabular}




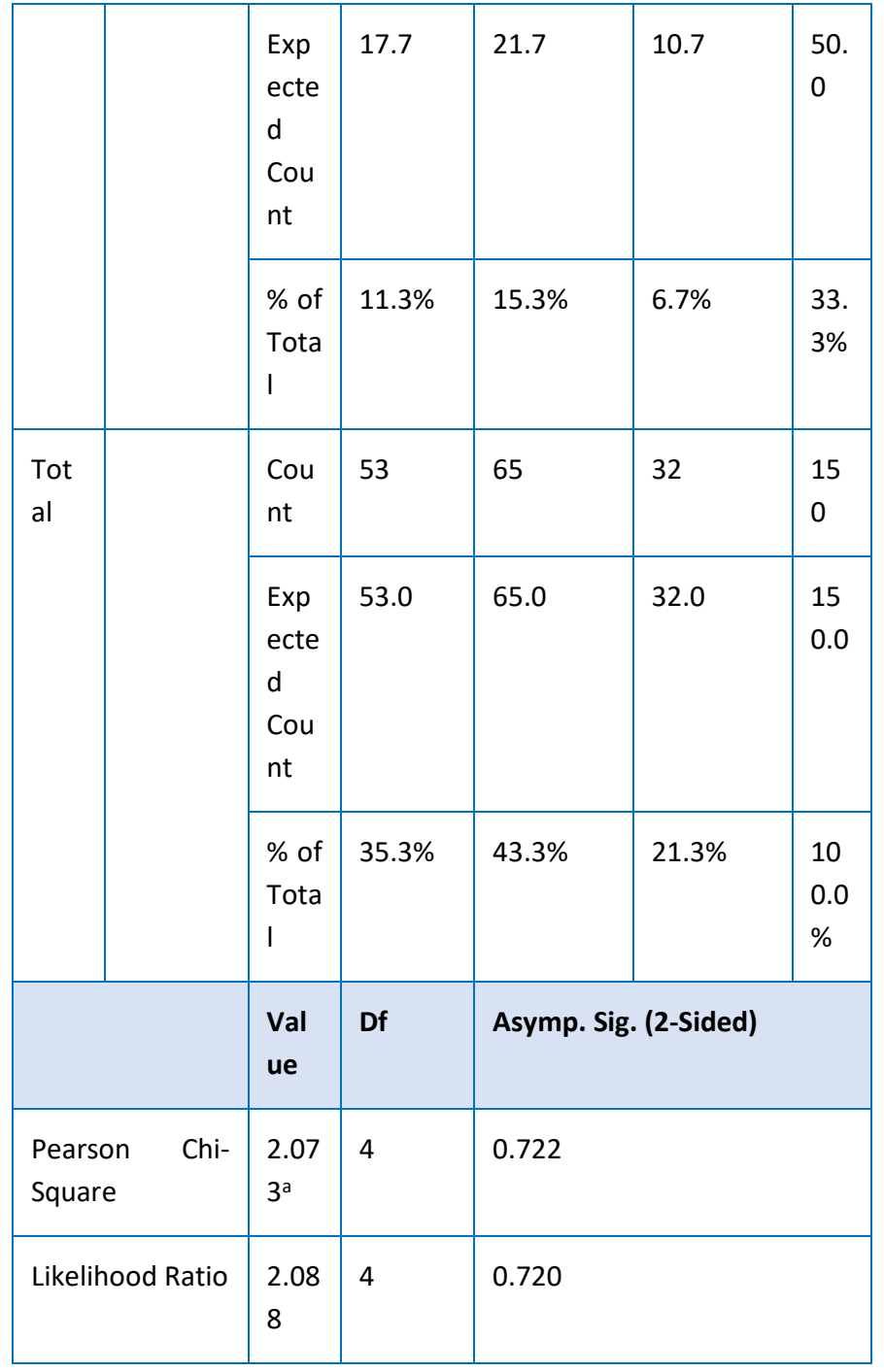

Chi-Square test was performed to see the Status of Kostha in different Prakruti. It was found that in the out of 150 individuals 35.3\% were Krura Kostha, 43.3\% were Madhyama Kostha and 21.3\% were Mrudu Kostha, among them in Vata Prakruti individuals Krura Kostha was dominant, in Pitta Prakruti and Kapha Prakruti individuals Madhyama Kostha was dominant at $p$ value 0.722 .

Table 2: Status of Quantity of stool in different Prakruti.

\begin{tabular}{|c|c|c|c|c|c|c|}
\hline & & & \multicolumn{3}{|c|}{ Quantity } & \multirow[t]{2}{*}{ Total } \\
\hline & & & $\begin{array}{l}\text { Krur } \\
\text { a } \\
\text { Kost } \\
\text { ha }\end{array}$ & $\begin{array}{l}\text { Madhya } \\
\text { ma } \\
\text { Kostha }\end{array}$ & $\begin{array}{l}\text { Mru } \\
d u \\
\text { Kost } \\
\text { ha }\end{array}$ & \\
\hline Prakr & Vatapradha & Count & 25 & 15 & 10 & 50 \\
\hline
\end{tabular}

\begin{tabular}{|c|c|c|c|c|c|c|}
\hline \multirow[t]{8}{*}{ iti } & \multirow[t]{2}{*}{$n a$} & $\begin{array}{l}\text { Expect } \\
\text { ed } \\
\text { Count }\end{array}$ & 21.0 & 18.0 & 11.0 & 50.0 \\
\hline & & $\begin{array}{l}\% \text { of } \\
\text { Total }\end{array}$ & $\begin{array}{l}16.7 \\
\%\end{array}$ & $10.0 \%$ & $6.7 \%$ & $\begin{array}{l}33.3 \\
\%\end{array}$ \\
\hline & \multirow{3}{*}{$\begin{array}{l}\text { Pittapradha } \\
\text { na }\end{array}$} & Count & 19 & 19 & 12 & 50 \\
\hline & & $\begin{array}{l}\text { Expect } \\
\text { ed } \\
\text { Count }\end{array}$ & 21.0 & 18.0 & 11.0 & 50.0 \\
\hline & & $\begin{array}{l}\% \text { of } \\
\text { Total }\end{array}$ & $\begin{array}{l}12.7 \\
\%\end{array}$ & $12.7 \%$ & $8.0 \%$ & $\begin{array}{l}33.3 \\
\%\end{array}$ \\
\hline & \multirow{3}{*}{$\begin{array}{l}\text { Kaphaprad } \\
\text { hana }\end{array}$} & Count & 19 & 20 & 11 & 50 \\
\hline & & $\begin{array}{l}\text { Expect } \\
\text { ed } \\
\text { Count }\end{array}$ & 21.0 & 18.0 & 11.0 & 50.0 \\
\hline & & $\begin{array}{l}\% \text { of } \\
\text { Total }\end{array}$ & $\begin{array}{l}12.7 \\
\%\end{array}$ & $13.3 \%$ & $7.3 \%$ & $\begin{array}{l}33.3 \\
\%\end{array}$ \\
\hline \multirow[t]{3}{*}{ Total } & & Count & 63 & 54 & 33 & 150 \\
\hline & & $\begin{array}{l}\text { Expect } \\
\text { ed } \\
\text { Count }\end{array}$ & 63.0 & 54.0 & 33.0 & $\begin{array}{l}150 . \\
0\end{array}$ \\
\hline & & $\begin{array}{l}\% \text { of } \\
\text { Total }\end{array}$ & $\begin{array}{l}42.0 \\
\%\end{array}$ & $36.0 \%$ & $\begin{array}{l}22.0 \\
\%\end{array}$ & $\begin{array}{l}100 . \\
0 \%\end{array}$ \\
\hline & & Value & df & \multicolumn{3}{|c|}{ Asymp. Sig. (2-sided) } \\
\hline \multicolumn{2}{|c|}{ Pearson Chi-Square } & $2.102^{\mathrm{a}}$ & 4 & \multicolumn{3}{|l|}{.717} \\
\hline \multicolumn{2}{|c|}{ Likelihood Ratio } & 2.093 & 4 & \multicolumn{3}{|l|}{.719} \\
\hline
\end{tabular}

Chi-Square test was performed to see the Status of quantity of stool in different Prakruti individuals andlt was found that out of 150 individuals in Vatapradhana Prakruti it was observed that majority individuals (16.7\%) were having Krura Kostha lakshana (habit of passing small quantity of stool ), in Pittapradhana Prakruti it was observed that majority individuals (12.7\%) were having Krura Kostha Lakshana (habit of passing small quantity of stool ) and also Madhyama Kostha Lakshana (habit of passing medium quantity of stool) and in Kaphapradhana Prakruti it was observed that 
majority individuals (13.3\%) were having Madhyama Kostha Lakshana (habit of passing medium quantity of stool) at $p$ value.717.

Table 3: Status of frequency of stool in different Prakruti.

\begin{tabular}{|c|c|c|c|c|c|c|}
\hline & & & \multicolumn{3}{|c|}{ Frequency } & \multirow[t]{2}{*}{ Total } \\
\hline & & & $\begin{array}{l}\text { Krur } \\
a \\
\text { Kost } \\
\text { ha }\end{array}$ & $\begin{array}{l}\text { Madhya } \\
\text { ma } \\
\text { Kostha }\end{array}$ & $\begin{array}{l}\text { Mru } \\
d u \\
\text { Kost } \\
\text { ha }\end{array}$ & \\
\hline \multirow{9}{*}{$\begin{array}{l}\text { Prakr } \\
\text { iti }\end{array}$} & \multirow{3}{*}{$\begin{array}{l}\text { Vatapradha } \\
\text { na }\end{array}$} & Count & 15 & 20 & 15 & 50 \\
\hline & & $\begin{array}{l}\text { Expect } \\
\text { ed } \\
\text { Count }\end{array}$ & 13.3 & 22.0 & 14.7 & 50.0 \\
\hline & & $\begin{array}{l}\% \text { of } \\
\text { Total }\end{array}$ & $\begin{array}{l}10.0 \\
\%\end{array}$ & $13.3 \%$ & $\begin{array}{l}10.0 \\
\%\end{array}$ & $\begin{array}{l}33.3 \\
\%\end{array}$ \\
\hline & \multirow{3}{*}{$\begin{array}{l}\text { Pittapradha } \\
\text { na }\end{array}$} & Count & 13 & 22 & 15 & 50 \\
\hline & & $\begin{array}{l}\text { Expect } \\
\text { ed } \\
\text { Count }\end{array}$ & 13.3 & 22.0 & 14.7 & 50.0 \\
\hline & & $\begin{array}{l}\% \text { of } \\
\text { Total }\end{array}$ & $8.7 \%$ & $14.7 \%$ & $\begin{array}{l}10.0 \\
\%\end{array}$ & $\begin{array}{l}33.3 \\
\%\end{array}$ \\
\hline & \multirow{3}{*}{$\begin{array}{l}\text { Kaphaprad } \\
\text { hana }\end{array}$} & Count & 12 & 24 & 14 & 50 \\
\hline & & $\begin{array}{l}\text { Expect } \\
\text { ed } \\
\text { Count }\end{array}$ & 13.3 & 22.0 & 14.7 & 50.0 \\
\hline & & $\begin{array}{l}\% \text { of } \\
\text { Total }\end{array}$ & $8.0 \%$ & $16.0 \%$ & $9.3 \%$ & $\begin{array}{l}33.3 \\
\%\end{array}$ \\
\hline \multirow[t]{3}{*}{ Total } & & Count & 40 & 66 & 44 & 150 \\
\hline & & $\begin{array}{l}\text { Expect } \\
\text { ed } \\
\text { Count }\end{array}$ & 40.0 & 66.0 & 44.0 & $\begin{array}{l}150 . \\
0\end{array}$ \\
\hline & & $\begin{array}{l}\% \text { of } \\
\text { Total }\end{array}$ & $\begin{array}{l}26.7 \\
\%\end{array}$ & $44.0 \%$ & $\begin{array}{l}29.3 \\
\%\end{array}$ & $\begin{array}{l}100 . \\
0 \%\end{array}$ \\
\hline & & Value & df & \multicolumn{3}{|c|}{ Asymp. Sig. (2-sided) } \\
\hline \multicolumn{2}{|c|}{ Pearson Chi-Square } & $.759^{a}$ & 4 & .944 & & \\
\hline
\end{tabular}

\begin{tabular}{|l|l|l|l|}
\hline Likelihood Ratio & .757 & 4 & .944 \\
\hline
\end{tabular}

Chi-Square test was performed to see the Status of frequency of stool in different Prakruti individuals and It was found that out of 150 individuals in Vatapradhana Prakruti (13.3\%), Pittapradhana Prakruti (14.7\%) and in Kaphapradhana Prakruti (16.0\%) majority individuals were having Madhyama Kostha Lakshana (habit of Passing Stool daily once or twice regularly) at $p$ value .944 .

Table 4: Status of Bowel clearance in different Prakruti

\begin{tabular}{|c|c|c|c|c|c|c|}
\hline & & & \multicolumn{3}{|c|}{ Evacuation } & \multirow{2}{*}{ Total } \\
\hline & & & $\begin{array}{l}\text { Krur } \\
a \\
\text { Kost } \\
\text { ha }\end{array}$ & $\begin{array}{l}\text { Madhya } \\
\text { ma } \\
\text { Kostha }\end{array}$ & $\begin{array}{l}\text { Mru } \\
\text { du } \\
\text { Kost } \\
\text { ha }\end{array}$ & \\
\hline \multirow{9}{*}{$\begin{array}{l}\text { Prakr } \\
\text { iti }\end{array}$} & \multirow{3}{*}{$\begin{array}{l}\text { Vatapradha } \\
\text { na }\end{array}$} & Count & 16 & 17 & 17 & 50 \\
\hline & & $\begin{array}{l}\text { Expect } \\
\text { ed } \\
\text { Count }\end{array}$ & 15.0 & 20.3 & 14.7 & 50.0 \\
\hline & & $\begin{array}{l}\% \text { of } \\
\text { Total }\end{array}$ & $\begin{array}{l}10.7 \\
\%\end{array}$ & $11.3 \%$ & $\begin{array}{l}11.3 \\
\%\end{array}$ & $\begin{array}{l}33.3 \\
\%\end{array}$ \\
\hline & \multirow{3}{*}{$\begin{array}{l}\text { Pittapradha } \\
\text { na }\end{array}$} & Count & 15 & 23 & 12 & 50 \\
\hline & & $\begin{array}{l}\text { Expect } \\
\text { ed } \\
\text { Count }\end{array}$ & 15.0 & 20.3 & 14.7 & 50.0 \\
\hline & & $\begin{array}{l}\% \text { of } \\
\text { Total }\end{array}$ & $\begin{array}{l}10.0 \\
\%\end{array}$ & $15.3 \%$ & $8.0 \%$ & $\begin{array}{l}33.3 \\
\%\end{array}$ \\
\hline & \multirow{3}{*}{$\begin{array}{l}\text { Kaphaprad } \\
\text { hana }\end{array}$} & Count & 14 & 21 & 15 & 50 \\
\hline & & $\begin{array}{l}\text { Expect } \\
\text { ed } \\
\text { Count }\end{array}$ & 15.0 & 20.3 & 14.7 & 50.0 \\
\hline & & $\begin{array}{l}\% \text { of } \\
\text { Total }\end{array}$ & $9.3 \%$ & $14.0 \%$ & $\begin{array}{l}10.0 \\
\%\end{array}$ & $\begin{array}{l}33.3 \\
\%\end{array}$ \\
\hline \multirow[t]{2}{*}{ Total } & & Count & 45 & 61 & 44 & 150 \\
\hline & & $\begin{array}{l}\text { Expect } \\
\text { ed }\end{array}$ & 45.0 & 61.0 & 44.0 & $\begin{array}{l}150 . \\
0\end{array}$ \\
\hline
\end{tabular}




\begin{tabular}{|l|l|l|l|l|l|}
\hline & Count & & & & \\
\cline { 2 - 6 } & $\begin{array}{l}\% \text { of } \\
\text { Total }\end{array}$ & $\begin{array}{l}30.0 \\
\%\end{array}$ & $40.7 \%$ & $\begin{array}{l}29.3 \\
\%\end{array}$ & $\begin{array}{l}100 . \\
0 \%\end{array}$ \\
\hline & Value & df & Asymp. Sig. (2-sided) \\
\hline Pearson Chi-Square & $1.915^{\mathrm{a}}$ & 4 & .751 & \\
\hline Likelihood Ratio & 1.947 & 4 & .745 \\
\hline
\end{tabular}

Chi-Square test was performed to see the Status of bowel clearance in different Prakruti individuals andlt was found that out of 150 individuals in Vatapradhana Prakruti it was observed that majority individuals (11.3\%) were having Madhyama Kostha Lakshana (feel satisfaction bowel clearance after defecation) and also Mrudu Kostha Lakshana (Occasionally feeling of satisfaction bowel clearance after defecation), Pittapradhana Prakruti (15.3\%) and in Kaphapradhana Prakruti (14.0\%) majority individuals were having Madhyama Kostha Lakshana (feel satisfaction bowel clearance after defecation). at p value.751.

Table 5: Status of Time for defecation in different Prakruti.

\begin{tabular}{|c|c|c|c|c|c|c|}
\hline & & & \multicolumn{3}{|l|}{ Time } & \multirow[t]{2}{*}{ Total } \\
\hline & & & $\begin{array}{l}\text { Krur } \\
a \\
\text { Kost } \\
\text { ha }\end{array}$ & $\begin{array}{l}\text { Madhya } \\
\text { ma } \\
\text { Kostha }\end{array}$ & $\begin{array}{l}\text { Mru } \\
d u \\
\text { Kost } \\
\text { ha }\end{array}$ & \\
\hline \multirow{6}{*}{$\begin{array}{l}\text { Prakr } \\
\text { iti }\end{array}$} & \multirow{3}{*}{$\begin{array}{l}\text { Vatapradha } \\
\text { na }\end{array}$} & Count & 20 & 19 & 11 & 50 \\
\hline & & $\begin{array}{l}\text { Expect } \\
\text { ed } \\
\text { Count }\end{array}$ & 17.3 & 20.0 & 12.7 & 50.0 \\
\hline & & $\begin{array}{l}\% \text { of } \\
\text { Total }\end{array}$ & $\begin{array}{l}13.3 \\
\%\end{array}$ & $12.7 \%$ & $7.3 \%$ & $\begin{array}{l}33.3 \\
\%\end{array}$ \\
\hline & \multirow{3}{*}{$\begin{array}{l}\text { Pittapradha } \\
\text { na }\end{array}$} & Count & 17 & 20 & 13 & 50 \\
\hline & & $\begin{array}{l}\text { Expect } \\
\text { ed } \\
\text { Count }\end{array}$ & 17.3 & 20.0 & 12.7 & 50.0 \\
\hline & & $\%$ of & 11.3 & $13.3 \%$ & $8.7 \%$ & 33.3 \\
\hline
\end{tabular}

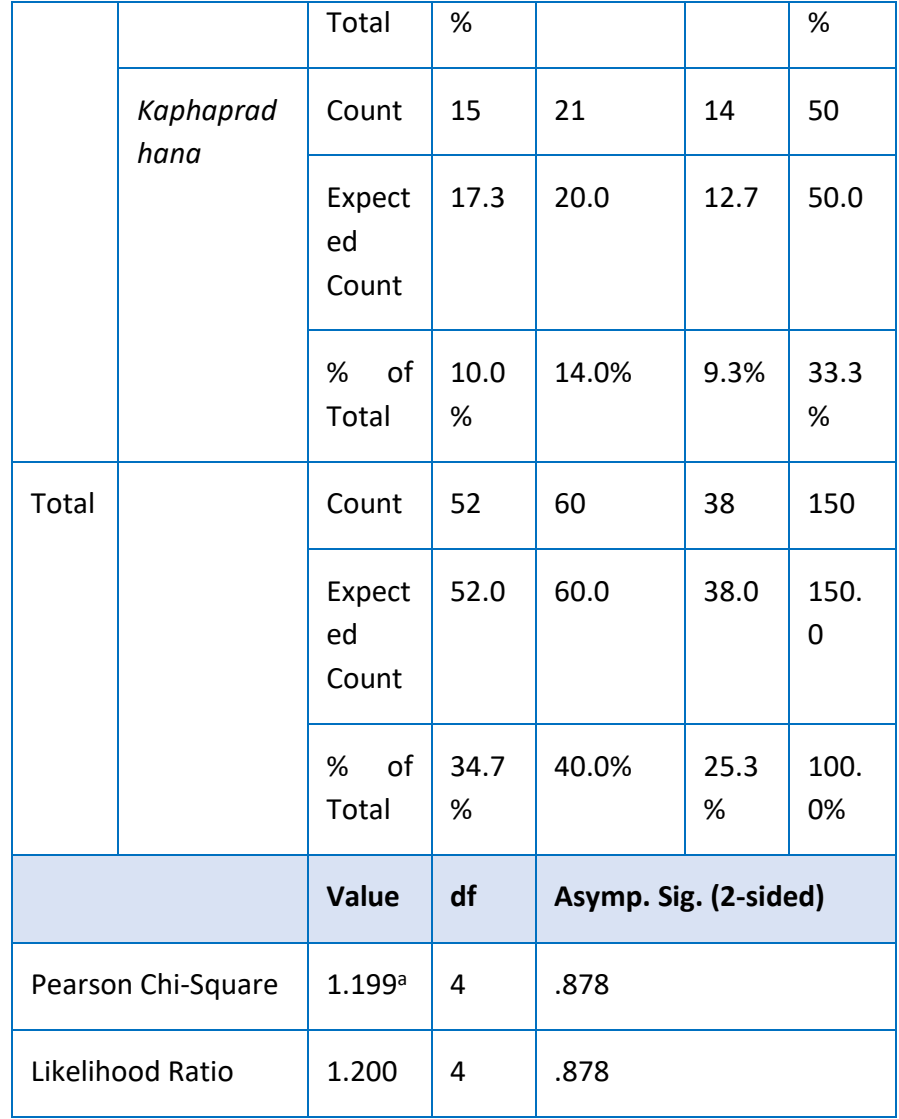

Chi-Square test was performed to see the Status of time for defecation in different Prakruti individuals and It was found that out of 150 individuals in Vatapradhana Prakruti it was observed that majority individuals (13.3\%) were having Krura Kostha Lakshana (require long time for defecation), Pittapradhana Prakruti (13.3\%) and in Kaphapradhana Prakruti (14.0\%) majority individuals were having Madhyama Kostha Lakshana (require little long time for defecation) at $p$ value .878 .

Table 6: Status of Colour of the stool in different Prakruti.

\begin{tabular}{|c|c|c|c|c|c|c|}
\hline & & & Colou & & & Total \\
\hline & & & $\begin{array}{l}\text { Krur } \\
a \\
\text { Kost } \\
\text { ha }\end{array}$ & $\begin{array}{l}\text { Madhya } \\
\text { ma } \\
\text { Kostha }\end{array}$ & $\begin{array}{l}\text { Mru } \\
d u \\
\text { Kost } \\
\text { ha }\end{array}$ & \\
\hline Prakr & Vatapradha & Count & 15 & 22 & 13 & 50 \\
\hline & & $\begin{array}{l}\text { Expect } \\
\text { ed } \\
\text { Count }\end{array}$ & 14.3 & 21.3 & 14.3 & 50.0 \\
\hline
\end{tabular}




\begin{tabular}{|c|c|c|c|c|c|c|}
\hline & & $\begin{array}{l}\% \text { of } \\
\text { Total }\end{array}$ & $\begin{array}{l}10.0 \\
\%\end{array}$ & $14.7 \%$ & $8.7 \%$ & $\begin{array}{l}33.3 \\
\%\end{array}$ \\
\hline & Pittapradha & Count & 14 & 24 & 12 & 50 \\
\hline & & $\begin{array}{l}\text { Expect } \\
\text { ed } \\
\text { Count }\end{array}$ & 14.3 & 21.3 & 14.3 & 50.0 \\
\hline & & $\begin{array}{l}\% \text { of } \\
\text { Total }\end{array}$ & $9.3 \%$ & $16.0 \%$ & $8.0 \%$ & $\begin{array}{l}33.3 \\
\%\end{array}$ \\
\hline & Kaphaprad & Count & 14 & 18 & 18 & 50 \\
\hline & & $\begin{array}{l}\text { Expect } \\
\text { ed } \\
\text { Count }\end{array}$ & 14.3 & 21.3 & 14.3 & 50.0 \\
\hline & & $\begin{array}{l}\% \text { of } \\
\text { Total }\end{array}$ & $9.3 \%$ & $12.0 \%$ & $\begin{array}{l}12.0 \\
\%\end{array}$ & $\begin{array}{l}33.3 \\
\%\end{array}$ \\
\hline Total & & Count & 43 & 64 & 43 & 150 \\
\hline & & $\begin{array}{l}\text { Expect } \\
\text { ed } \\
\text { Count }\end{array}$ & 43.0 & 64.0 & 43.0 & $\begin{array}{l}150 . \\
0\end{array}$ \\
\hline & & $\begin{array}{l}\% \text { of } \\
\text { Total }\end{array}$ & $\begin{array}{l}28.7 \\
\%\end{array}$ & $42.7 \%$ & $\begin{array}{l}28.7 \\
\%\end{array}$ & $\begin{array}{l}100 . \\
0 \%\end{array}$ \\
\hline & & Value & df & Asymp. & (2-sid & \\
\hline Pears & Chi-Square & $2.363^{\mathrm{a}}$ & 4 & .669 & & \\
\hline Likelir & od Ratio & 2.335 & 4 & .674 & & \\
\hline
\end{tabular}

Chi-Square test was performed to see the Status of Colour of the stool in different Prakruti individuals and It was found that out of 150 individuals in Vatapradhana Prakruti (14.7\%) and in Pittapradhana Prakruti (16.0\%) it was observed that majority individuals were having Madhyama Kostha Lakshana (Sometimes observed discolouration of the stool) and in Kaphapradhana Prakruti it was observed that majority individuals (12.0\%) were having Madhyama Kostha Lakshana (Sometimes observed discolouration of the stool) and also Mrudu Kostha Lakshana (Observed no changes in colour of the stool) at $p$ value.669.
Table 7: Status of Smell in the stool in different Prakruti.

\begin{tabular}{|c|c|c|c|c|c|c|}
\hline & & & \multicolumn{3}{|c|}{ Smell } & \multirow[t]{2}{*}{ Total } \\
\hline & & & $\begin{array}{l}\text { Krur } \\
\text { a } \\
\text { Kost } \\
\text { ha }\end{array}$ & $\begin{array}{l}\text { Madhya } \\
\text { ma } \\
\text { Kostha }\end{array}$ & $\begin{array}{l}\text { Mru } \\
\text { du } \\
\text { Kost } \\
\text { ha }\end{array}$ & \\
\hline \multirow{9}{*}{$\begin{array}{l}\text { Prakr } \\
\text { iti }\end{array}$} & \multirow{3}{*}{$\begin{array}{l}\text { Vatapradha } \\
\text { na }\end{array}$} & Count & 18 & 14 & 18 & 50 \\
\hline & & $\begin{array}{l}\text { Expect } \\
\text { ed } \\
\text { Count }\end{array}$ & 16.7 & 18.3 & 15.0 & 50.0 \\
\hline & & $\begin{array}{l}\% \text { of } \\
\text { Total }\end{array}$ & $\begin{array}{l}12.0 \\
\%\end{array}$ & $9.3 \%$ & $\begin{array}{l}12.0 \\
\%\end{array}$ & $\begin{array}{l}33.3 \\
\%\end{array}$ \\
\hline & \multirow{3}{*}{$\begin{array}{l}\text { Pittapradha } \\
\text { na }\end{array}$} & Count & 15 & 22 & 13 & 50 \\
\hline & & $\begin{array}{l}\text { Expect } \\
\text { ed } \\
\text { Count }\end{array}$ & 16.7 & 18.3 & 15.0 & 50.0 \\
\hline & & $\begin{array}{l}\% \text { of } \\
\text { Total }\end{array}$ & $\begin{array}{l}10.0 \\
\%\end{array}$ & $14.7 \%$ & $8.7 \%$ & $\begin{array}{l}33.3 \\
\%\end{array}$ \\
\hline & \multirow{3}{*}{$\begin{array}{l}\text { Kaphaprad } \\
\text { hana }\end{array}$} & Count & 17 & 19 & 14 & 50 \\
\hline & & $\begin{array}{l}\text { Expect } \\
\text { ed } \\
\text { Count }\end{array}$ & 16.7 & 18.3 & 15.0 & 50.0 \\
\hline & & $\begin{array}{l}\% \text { of } \\
\text { Total }\end{array}$ & $\begin{array}{l}11.3 \\
\%\end{array}$ & $12.7 \%$ & $9.3 \%$ & $\begin{array}{l}33.3 \\
\%\end{array}$ \\
\hline \multirow[t]{3}{*}{ Total } & & Count & 50 & 55 & 45 & 150 \\
\hline & & $\begin{array}{l}\text { Expect } \\
\text { ed } \\
\text { Count }\end{array}$ & 50.0 & 55.0 & 45.0 & $\begin{array}{l}150 . \\
0\end{array}$ \\
\hline & & $\begin{array}{l}\% \text { of } \\
\text { Total }\end{array}$ & $\begin{array}{l}33.3 \\
\%\end{array}$ & $36.7 \%$ & $\begin{array}{l}30.0 \\
\%\end{array}$ & $\begin{array}{l}100 . \\
0 \%\end{array}$ \\
\hline & & Value & df & \multicolumn{3}{|c|}{ Asymp. Sig. (2-sided) } \\
\hline \multicolumn{2}{|c|}{ Pearson Chi-Square } & $2.995^{a}$ & 4 & .559 & & \\
\hline \multicolumn{2}{|c|}{ Likelihood Ratio } & 3.023 & 4 & .554 & & \\
\hline
\end{tabular}

Chi-Square test was performed to see the Status of smell in the stool in different Prakruti individuals andlt 
was found that out of 150 individuals in Vatapradhana Prakruti it was observed that majority individuals (12.0\%) were having Krura Kostha Lakshana (observed foul smell in the stool) and also Mrudu Kostha Lakshana (Sometimes observed foul smell in the stool) and in Pittapradhana Prakruti (14.7\%), Kaphapradhana Prakruti (12.7\%) majority individuals were having Madhyama Kostha Lakshana (not observed foul smell in the stool) at p value.559.

Table 8: Status of straining for defecation while passing the stool in different Prakruti.

\begin{tabular}{|c|c|c|c|c|c|c|}
\hline & & & \multicolumn{3}{|c|}{ Symptoms } & \multirow[t]{2}{*}{ Total } \\
\hline & & & $\begin{array}{l}\text { Krur } \\
a \\
\text { Kost } \\
\text { ha }\end{array}$ & $\begin{array}{l}\text { Madhya } \\
\text { ma } \\
\text { Kostha }\end{array}$ & $\begin{array}{l}\text { Mru } \\
d u \\
\text { Kost } \\
\text { ha }\end{array}$ & \\
\hline \multirow{9}{*}{$\begin{array}{l}\text { Prakr } \\
\text { iti }\end{array}$} & \multirow{3}{*}{$\begin{array}{l}\text { Vatapradha } \\
\text { na }\end{array}$} & Count & 20 & 19 & 11 & 50 \\
\hline & & $\begin{array}{l}\text { Expect } \\
\text { ed } \\
\text { Count }\end{array}$ & 17.3 & 20.0 & 12.7 & 50.0 \\
\hline & & $\begin{array}{l}\% \text { of } \\
\text { Total }\end{array}$ & $\begin{array}{l}13.3 \\
\%\end{array}$ & $12.7 \%$ & $7.3 \%$ & $\begin{array}{l}33.3 \\
\%\end{array}$ \\
\hline & \multirow{3}{*}{$\begin{array}{l}\text { Pittapradha } \\
\text { na }\end{array}$} & Count & 17 & 20 & 13 & 50 \\
\hline & & $\begin{array}{l}\text { Expect } \\
\text { ed } \\
\text { Count }\end{array}$ & 17.3 & 20.0 & 12.7 & 50.0 \\
\hline & & $\begin{array}{l}\% \text { of } \\
\text { Total }\end{array}$ & $\begin{array}{l}11.3 \\
\%\end{array}$ & $13.3 \%$ & $8.7 \%$ & $\begin{array}{l}33.3 \\
\%\end{array}$ \\
\hline & \multirow{3}{*}{$\begin{array}{l}\text { Kaphaprad } \\
\text { hana }\end{array}$} & Count & 15 & 21 & 14 & 50 \\
\hline & & $\begin{array}{l}\text { Expect } \\
\text { ed } \\
\text { Count }\end{array}$ & 17.3 & 20.0 & 12.7 & 50.0 \\
\hline & & $\begin{array}{l}\% \text { of } \\
\text { Total }\end{array}$ & $\begin{array}{l}10.0 \\
\%\end{array}$ & $14.0 \%$ & $9.3 \%$ & $\begin{array}{l}33.3 \\
\%\end{array}$ \\
\hline \multirow[t]{2}{*}{ Total } & & Count & 52 & 60 & 38 & 150 \\
\hline & & $\begin{array}{l}\text { Expect } \\
\text { ed } \\
\text { Count }\end{array}$ & 52.0 & 60.0 & 38.0 & $\begin{array}{l}150 . \\
0\end{array}$ \\
\hline
\end{tabular}

\begin{tabular}{|l|l|l|l|l|l|}
\hline & $\begin{array}{l}\% \text { of } \\
\text { Total }\end{array}$ & $\begin{array}{l}34.7 \\
\%\end{array}$ & $40.0 \%$ & $\begin{array}{l}25.3 \\
\%\end{array}$ & $\begin{array}{l}100 . \\
0 \%\end{array}$ \\
\hline & Value & df & Asymp. Sig. (2-sided) \\
\hline Pearson Chi-Square & $1.199^{a}$ & 4 & .878 \\
\hline Likelihood Ratio & 1.200 & 4 & .878 \\
\hline
\end{tabular}

Chi-Square test was performed to see the status of straining for defecation while passing the stool in different Prakrutiindividuals andlt was found that out of 150 individuals in VatapradhanaPrakruti it was observed that majority individuals (13.3\%) were having KruraKosthaLakshana (requires straining for defecation) and in PittapradhanaPrakruti (13.3\%), KaphapradhanaPrakruti (14.0\%) majority individuals were having Madhyama KosthaLakshana (requires minimum stress for defecation) at $p$ value. 878 .

Table 9: Status of Application of Laxative / Purgative for proper defecation in different Prakruti.

\begin{tabular}{|c|c|c|c|c|c|c|}
\hline & & & \multicolumn{3}{|c|}{ Purgatives } & \multirow[t]{2}{*}{ Total } \\
\hline & & & $\begin{array}{l}\text { Krur } \\
a \\
\text { Kost } \\
\text { ha }\end{array}$ & $\begin{array}{l}\text { Madhya } \\
\text { ma } \\
\text { Kostha }\end{array}$ & $\begin{array}{l}\text { Mru } \\
d u \\
\text { Kost } \\
\text { ha }\end{array}$ & \\
\hline \multirow{8}{*}{$\begin{array}{l}\text { Prakr } \\
\text { iti }\end{array}$} & \multirow{3}{*}{$\begin{array}{l}\text { Vatapradha } \\
\text { na }\end{array}$} & Count & 16 & 22 & 12 & 50 \\
\hline & & $\begin{array}{l}\text { Expect } \\
\text { ed } \\
\text { Count }\end{array}$ & 16.7 & 22.7 & 10.7 & 50.0 \\
\hline & & $\begin{array}{l}\% \text { of } \\
\text { Total }\end{array}$ & $\begin{array}{l}10.7 \\
\%\end{array}$ & $14.7 \%$ & $8.0 \%$ & $\begin{array}{l}33.3 \\
\%\end{array}$ \\
\hline & \multirow{3}{*}{$\begin{array}{l}\text { Pittapradha } \\
\text { na }\end{array}$} & Count & 16 & 24 & 10 & 50 \\
\hline & & $\begin{array}{l}\text { Expect } \\
\text { ed } \\
\text { Count }\end{array}$ & 16.7 & 22.7 & 10.7 & 50.0 \\
\hline & & $\begin{array}{l}\% \text { of } \\
\text { Total }\end{array}$ & $\begin{array}{l}10.7 \\
\%\end{array}$ & $16.0 \%$ & $6.7 \%$ & $\begin{array}{l}33.3 \\
\%\end{array}$ \\
\hline & \multirow{2}{*}{$\begin{array}{l}\text { Kaphaprad } \\
\text { hana }\end{array}$} & Count & 18 & 22 & 10 & 50 \\
\hline & & $\begin{array}{l}\text { Expect } \\
\text { ed } \\
\text { Count }\end{array}$ & 16.7 & 22.7 & 10.7 & 50.0 \\
\hline
\end{tabular}




\begin{tabular}{|l|l|l|l|l|l|l|}
\hline & $\begin{array}{l}\% \text { of } \\
\text { Total }\end{array}$ & $\begin{array}{l}12.0 \\
\%\end{array}$ & $14.7 \%$ & $6.7 \%$ & $\begin{array}{l}33.3 \\
\%\end{array}$ \\
\hline \multirow{2}{*}{ Total } & Count & 50 & 68 & 32 & 150 \\
\cline { 2 - 7 } & $\begin{array}{l}\text { Expect } \\
\text { ed } \\
\text { Count }\end{array}$ & 50.0 & 68.0 & 32.0 & $\begin{array}{l}150 . \\
0\end{array}$ \\
\cline { 2 - 6 } & $\begin{array}{l}\% \text { of } \\
\text { Total }\end{array}$ & $\begin{array}{l}33.3 \\
\%\end{array}$ & $45.3 \%$ & $\begin{array}{l}21.3 \\
\%\end{array}$ & $\begin{array}{l}100 . \\
0 \%\end{array}$ \\
\hline Pearson Chi-Square & Value & df & Asymp. Sig. (2-sided) \\
\hline Likelihood Ratio & 4 & \multicolumn{2}{|l|}{.971} & \multicolumn{2}{l|}{.972} \\
\hline
\end{tabular}

Chi-Square test was performed to see the Status of Application of Laxative / Purgative for proper defecation in different Prakruti individuals andlt was found that out of 150 individuals in Vatapradhana Prakruti (14.7\%), Pittapradhana Prakruti (16.0\%) and in Kaphapradhana Prakruti (14.7\%) majority individuals were having Madhyama Kostha Lakshana (requires medium dose of Purgatives or laxatives for proper defecation) at $p$ value.971.

\section{DISCUSSION}

\section{Status of Kostha in different Prakruti}

In the study it revealed that among 150 individuals 53 individuals were having Krura Kostha, 65 individuals were having Madhyama Kostha and 32 individuals were having MruduKostha.Majority of them were having Madhyama Kostha. This might be due to the Ahara \& Vihara of the individuals and also due to the effect of Varsha Rutu (season of aggravation of the Vata) because of Ruksha (Dry) and Khara (Rough) Guna of Vata will in turn have its influence on Kostha.

Among 150 individuals in Vata Prakruti individuals Krura Kostha was dominant, this might be due to the unpredictable and inconsistent nature of Vata the fire too keeps varying, and sometimes it is good and sometimes not. The digestion of food is also sometimes good and sometimes worst and the status of Kostha also follows the status of Agni. The Kostha is also Vishama when the Agni digests the food properly, the bowel movements will be good when the digestion of the food is bad, the bowel movements will be bad. Thus the Krura Kostha is observed in Vata Prakruti individuals. ${ }^{[59]}$

Among 150 individuals in Pitta Prakruti individuals Madhyama Kostha was dominantal though Theekshanagni is predominant in Pitta Prakruti individuals, thus Pitta and Agni are same elements and Pitta is predominantly associates with Agni of the individuals. The fire digests the food quickly and makes Mrudu Kostha, the alteration of status of Kostha might be due to the Ahara \& Vihara of the individuals and also due to the effect of Varsha Rutu (season of aggravation of the Vata), Ruksha (Dry) and Khara (Rough) Guna of Vata will in turn have its influence on Kostha.

Among 150 individuals in Kapha Prakruti individuals Madhyama Kostha was dominant this might be due the Mandagni is predominant in Kapha Prakruti individuals; the digestion of this type of Agni will be mild \& moderate. Kapha acts like a buffer and does not allow the Vata \& Pitta to go to the surge. Therefore fire is mild \& moderate type and this will impacts upon the status of Kostha.

\section{Status of Quantity of stool in different Prakruti}

Among 150 individuals it was found that in Vatapradhana Prakruti majority individuals were having Krura Kostha Lakshana (habit of passing small quantity of stool), this might be due to the inconsistent nature of Vata and also because of theVishamagni influences upon the bowel quantity of the individuals.

Among 150 individuals it was found that in Pittapradhana Prakruti majority individuals were having Krura Kostha Lakshana (habit of passing small quantity of stool) and also Madhyama Kostha Lakshana (habit of passing medium quantity of stool) this might be due the Ahara \& Vihara of the individuals and also due to the effect of Varsha Rutu (season of aggravation of the Vata) will in turn have its influence on bowel quantity of the individual.

Among 150 individuals it was found that in Kaphapradhana Prakruti majority individuals were 
having Madhyama Kostha Lakshana (habit of passing medium quantity of stool). This might be due to because of the association of Kapha and Agni makes the bowel quantity is moderate.

\section{Status of Frequency of stool in different Prakruti}

Among 150 individuals it was found that in Vatapradhana Prakruti, Pittapradhana Prakruti and in Kaphapradhana Prakruti majority individuals were having Madhyama Kostha Lakshana (habit of Passing Stool daily once or twice regularly) this might be due to because of the Ahara \& Vihara of the individuals which alters the status of Kostha Lakshana in Vatapradhana Prakruti, Pittapradhana Prakruti individuals and it is not natural, it is a part of pathology.

\section{Status of Bowel clearance in different Prakruti}

Among 150 individuals it was found that in Vatapradhana Prakruti majority individuals were having Madhyama Kostha Lakshana (feel satisfaction bowel clearance after defecation) and also Mrudu Kostha Lakshana (Occasionally feeling of satisfaction bowel clearance after defecation), Pittapradhana Prakruti and in Kaphapradhana Prakruti majority individuals were having Madhyama Kostha Lakshana (feel satisfaction bowel clearance after defecation) this might be due to because of the Ahara \& Vihara of the individuals which is Kapha in nature which is predominant of the Jala \& Prithvi Mahabhuta and the combination is usually heavy and facilitates the gravitation and this enables the satisfaction in the bowel clearance after defecation.

\section{Status of Time for defecation in different Prakruti}

Among 150 individuals it was found that in Vatapradhana Prakruti majority individuals were having Krura Kostha Lakshana (requires long time for defecation) this might be due to because of Ruksha (Dry) and Khara (Rough) Guna of Vata in Rutu the watery content of the stool gets almost absorbed this makes the stools stub born and pellet form, due to less moisture or no moisture content gets adhered to the walls of intestine, makes the stool passing difficulty and requires long time for defecation and in
Pittapradhana Prakruti, Kaphapradhana Prakruti individuals majority individuals were having Madhyama Kostha Lakshana (requires little long time for defecation) this might be due to because of Sthira (Stable), Manda (Slow) and Pichhila (Sticky) Guna of Kapha prevents the stool from getting cleared with accurate easiness so, requiress little long time for defecation in individual.

\section{Status of Colour of the stool in different Prakruti}

Among 150 individuals in Vatapradhana Prakruti and in Pittapradhana Prakruti majority individuals were having Madhyama Kostha Lakshana (Sometimes observed discolouration of the stool) and in Kaphapradhana Prakruti majority individuals were having Madhyama Kostha Lakshana (Sometimes observed discolouration of the stool) and also Mrudu Kostha Lakshana (Observed no changes in colour of the stool) this might be due to because of the Ahara of the individuals which alters the status of Kostha Lakshana.

\section{Status of Smell in the stool in different Prakruti}

Among 150 individuals it was found that in Vatapradhana Prakruti that majority individuals were having Krura Kostha Lakshana (observed foul smell in the stool) and also Mrudu Kostha Lakshana (Sometimes observed foul smell in the stool). This might be due to the unpredictable and inconsistent nature of Vishamagni, sometimes it is good and sometimes not. The digestion of food is also sometimes good and sometimes worst and the status of Kostha also follows the status of Agni when the digestion of the food is improper, foul smell was observed in the stool because of Ama, thus the Krura Kostha Lakshana is observed in Vata Prakruti individuals and in Pittapradhana Prakruti majority individuals were having Madhyama Kostha Lakshana (not observed foul smell in the stool). This might be due to the Theekshanagni is predominant in Pitta Prakruti individuals, thus Pitta and Agni are same elements and Pitta is predominantly associates with Agni of the individuals. The fire digests the food properly so foul smell was not observed in the stool and in Kaphapradhana Prakruti majority individuals 
were having Madhyama Kostha Lakshana (not observed foul smell in the stool) this might be due to because of the Ahara \& Vihara of the individuals which alters the status of Kostha Lakshana.

Status of straining for defecation while passing the stool in different Prakruti

Among 150 individuals it was found that in Pittapradhana Prakruti, Kaphapradhana Prakruti majority individuals were having Madhyama Kostha Lakshana (requires minimum stress for defecation) this might be due to because of Sthira (Stable), Manda (Slow) and Pichhila (Sticky) Guna of Kapha prevents the stool from getting cleared with accurate easiness so, minimum stress is required for defecation in individual and in Vatapradhana Prakruti majority individuals were having Krura Kostha Lakshana (requires straining for defecation) this might be due to because of Ruksha (Dry) and Khara (Rough) Guna of Vata in Rutu the watery content of the stool gets almost absorbed this makes the stools stub born and pellet form, due to less moisture or no moisture content gets adhered to the walls of intestine, and makes the stool passing difficulty in Vatapradhana Prakruti individuals and also alters the status of Kostha Lakshana in Pittapradhana Prakruti individuals.

Status of Application of Laxative / Purgative for proper defecation in different Prakruti

Among 150 individuals it was found that in Vatapradhana Prakruti, Pittapradhana Prakruti and in Kaphapradhana Prakruti majority individuals were having Madhyama Kostha Lakshana (requires medium dose of Purgatives or laxatives for proper defecation) this might be due to because of the Sheeta (Cold), Guru (Heavy), Manda (Slow) Guna of the Rutu prevents the stools from getting cleared with accurate easiness and requires medium dose of Purgatives or laxatives for proper defecation. Which alters the status of Kostha Lakshana in Vatapradhana Prakruti, Pittapradhana Prakruti individuals and it is not natural, it will not be a part of Prakruti, it will be a Vikriti.

\section{CONCLUSION}

Based upon the study the following conclusions can be drawn; The predominance of Krura Kostha was found evident in Vata Pradhana Prakruthi individuals. The predominance of Madhyama Kostha was found evident in Pitta Pradhana Prakruthi and Kapha Pradhana Prakruthi individuals. Ahara \& Vihara of the individuals have influence on the status of Kostha. The Vata which have been aggravated in Varsha Rutu have great influence on the status of Kostha. Koshta concepts helps to understand selection of appropriate and specific treatment in a given disease. Advices can be given regarding do's and don'ts of Ahara and Vihara based upon the status of Kostha of individuals to prevent diseases and for the promotion of health.

\section{REFERENCES}

1. P.V. Sharma. Susruta Samhita with Nibandha sangraha commentary of Dalhana. Reprint ed. Varanasi (India): Chaukambha Sanskrit Sansthan; 2005. p. 162.

2. Acharya JT. Charaka Samhita with Ayurveda Dipika commentary of ChakrapaniDatta. Reprint ed. Varanasi (India): Chaukambha Orientalia; 2007. p. 52.

3. Acharya JT. Charaka Samhita with Ayurveda Dipika commentary of ChakrapaniDatta. Reprint ed. Varanasi (India): ChaukambhaOrientalia; 1994. p. 277.

4. Paradara HSS. Ashtanga Hrudaya with Sarvanga sundara commentary of Arunadatta and Ayurveda rasayana commentary of Hemadri. 9th ed. Varanasi (India): Chaukambha Orientalia; 2000. p. 7 .

5. Paradara HSS. Ashtanga Hrudaya with Sarvanga sundara commentary of Arunadatta and Ayurveda rasayana commentary of Hemadri. 9th ed. Varanasi (India): Chaukambha Orientalia; 2000. p. 8-9.

6. Dr. Anant Ram Sharma, edited with 'susruta vimarsini' Hindi commentary. (Ed.). Susruta samhita, maharshi susruta. Chikitsastan; 


\section{ISSN: 2456-3110}

\section{ORIGINAL ARTICLE}

Jan-Feb 2020 vamanvirechanasadhyoupadrava-adhyaya:

Chapter 33. verse no.20. Varanasi : Chukhambha prakashan, 2010 ; page no.427.

7. Acharya JT. Charaka Samhita with Ayurveda Dipika commentary of Chakrapani Datta. Reprint ed. Varanasi (India): Chaukambha Orientalia; 2007. p. 255.

8. Paradara HSS. Ashtanga Hrudaya with Sarvanga sundara commentary of Arunadatta and Ayurveda rasayana commentary of Hemadri. $9^{\text {th }}$ ed. Varanasi (India): Chaukambha Orientalia; 2005. p.39-43.

9. Paradara HSS. Ashtanga Hrudaya with Sarvanga sundara commentary of Arunadatta and Ayurveda rasayana commentary of Hemadri. 9th ed. Varanasi (India): Chaukambha Orientalia; 2005. p.37.
10. Acharya JT. Susrutha Samhita with Nibandha sangraha commentary of Dalhana. Reprint ed. Varanasi (India): Chaukambha Sanskrit Sansthan; 2009. p. 24.

11. Acharya JT. Susrutha Samhita with Nibandha sangraha commentary of Dalhana. Reprint ed. Varanasi (India): Chaukambha Sanskrit Sansthan; 2009. p. 29.

How to cite this article: Divyasree $\mathrm{CH}$, Sunil Govind Kahalekhar, Rashmi NM. A study on the status of Kostha in Sravana and Bhadrapada Masa in different Prakruti. J Ayurveda Integr Med Sci 2020;1:52-62. http://dx.doi.org/10.21760/jaims.5.1.10

Source of Support: Nil, Conflict of Interest: None declared.

Copyright (C) 2020 The Author(s); Published by Maharshi Charaka Ayurveda Organization, Vijayapur (Regd). This is an open-access article distributed under the terms of the Creative Commons Attribution License (http://creativecommons.org/licenses/by/4.0), which permits unrestricted use, distribution, and reproduction in any medium, provided the original work is properly cited. 